\title{
A Multiple-Layer Finite-Element Model of the Surface EMG Signal
}

\author{
Madeleine M. Lowery*, Member, IEEE, Nikolay S. Stoykov, Member, IEEE, Allen Taflove, Fellow, IEEE, and \\ Todd A. Kuiken, Member, IEEE
}

\begin{abstract}
The effect of skin, muscle, fat, and bone tissue on simulated surface electromyographic (EMG) signals was examined using a finite-element model. The amplitude and frequency content of the surface potential were observed to increase when the outer layer of a homogeneous muscle model was replaced with highly resistive skin or fat tissue. The rate at which the surface potential decreased as the fiber was moved deeper within the muscle also increased. Similarly, the rate at which the surface potential decayed around the surface of the model, for a constant fiber depth, increased. When layers of subcutaneous fat of increasing thickness were then added to the model, EMG amplitude, frequency content, and the rate of decay of the surface EMG signal around the limb decreased, due to the increased distance between the electrodes and the active fiber. The influence of bone on the surface potential was observed to vary considerably, depending on its location. When located close to the surface of the volume conductor, the surface EMG signal between the bone and the source and directly over the bone increased, accompanied by a slight decrease on the side of the bone distal to the active fiber. The results emphasize the importance of distinguishing between the effects of material properties and the distance between source and electrode when considering the influence of subcutaneous tissue, and suggest possible distortions in the surface EMG signal in regions where a bone is located close to the skin surface.
\end{abstract}

Index Terms-Finite-element analysis, mathematical model, surface EMG.

\section{INTRODUCTION}

$\mathbf{M}$ ODELING of the surface electromyographic (EMG) signal has proved to be a valuable tool in helping to interpret and understand what is a highly complex signal. A wide range of models has been presented over recent decades, from simulations of single fiber and motor unit action potentials to the complex interference patterns typical of voluntary

\footnotetext{
Manuscript received July 27, 2001; revised December 31, 2001. This work was supported in part by the Whitaker Foundation under a biomedical engineering research grant, in part by the National Institute of Child and Human Development under Grant \#1 K08HD01224-01 A1, and in part by the National Institute of Disability and Rehabilitation Research under Grant \#H133G990074-00. Asterisk indicates corresponding author.

*M. M. Lowery is with the Rehabilitation Institute of Chicago and in the Department of Physical Medicine and Rehabilitation, Northwestern University, Chicago, IL 60611 USA (e-mail: m-lowery@ northwestern.edu).

N. S. Stoykov is with the Rehabilitation Institute of Chicago and in the Department of Physical Medicine and Rehabilitation, Northwestern University Medical School, Chicago, IL 60611 USA.

A. Taflove is with the Department of Electrical and Computer Engineering, Northwestern University, Evanston, IL 60208 USA.

T. A. Kuiken is with the Rehabilitation Institute of Chicago and in the Department of Physical Medicine and Rehabilitation, Northwestern University Medical School, Chicago, IL 60611 USA; and the Electrical and Computer Engineering Department, Northwestern University, Evanston, IL 60208 USA.

Publisher Item Identifier S 0018-9294(02)03990-3.
}

contractions. These models have been predominantly based on analytical solutions for muscle fibers located in either finite or infinite volume conductors [1]-[6]. Analytical models, however, are limited to simple cylindrical or elliptical geometries and become increasingly difficult to handle as additional layers are incorporated. To simulate more physiological limb geometries, numerical methods such as boundary or finite-element (FE) methods must be employed [7], [8]. Although the application of numerical methods to surface EMG has been limited to date, boundary and finite-element models previously presented [8]-[10], illustrate how numerical methods may be successfully used to model the electrical fields around active nerve and muscle fibers.

To date, a limited number of models have considered the finite nature of the volume conductor in which the muscle fibers lie [3]-[6], [11]. Of these, only the models of Gootzen et al., [3] and Roeleveld et al., [5] have incorporated multiple layers of fat and skin tissue, although fat and skin layers have been included in a recent hemi-space model for planar geometries [12]. The highly resistive skin and adipose tissues that lie between the electrode and muscle are commonly neglected. It also remains unclear how the presence of a bone can affect the EMG signal detected at the skin surface. Studies by Wilson et al., [13] on the effect of a centrally located bone on nerve action potentials and by Schneider et al., [8] on the presence of small regions of inhomogeneous tissue suggest that the presence of a major inhomogeneity such as bone may considerably distort the potential distribution on the skin surface.

To examine these issues in further detail, a finite-element model of the surface EMG signal is presented in this paper. Using the model, the effect of inhomogeneous tissues on the surface EMG signal is examined. Tissue conductivity values described in a recent review of the dielectric properties of a range of biological tissues are incorporated in the model [14]-[16]. Effects due to highly resistive skin, adipose tissue, and bone are examined with the aim of establishing how each physiological parameter affects the surface EMG signal. In particular, the influence of subcutaneous tissue thickness and superficial bone on the rate of decay of the EMG signal around the limb was examined. The rate at which the surface potential decays with angular displacement from the source has important implications in the study of surface EMG crosstalk. While previous experimental studies have reported variations in crosstalk with subcutaneous tissue thickness in the upper arm [17], [18] and with proximity to the tibial bone [19], the reasons behind these observations remain unclear. Model simulations present an opportunity to examine how physical variations such as these affect EMG selec- 
tivity. For simplicity, only single fiber action potentials are considered here. Although the influence of fiber distribution, motor unit type and location, motor unit firing statistics and synchronization must be considered when interpreting voluntary surface EMG signals, the single fiber model is a convenient method of exploring issues related to volume conduction without the compounding effects related to motor control.

\section{METHODS}

\section{A. Finite-Element Model}

The finite-element method was used to calculate the potential in an idealized cylindrical limb due to a muscle fiber located within the muscle tissue. The conducting volume was divided into sets of linear tetrahedral and quadrilateral elements. Each element set corresponded to a different tissue type within the model. Elements of a very small size were used to mesh the areas surrounding the regions of interest to facilitate a high level of accuracy where required, while a coarser resolution was used elsewhere in the model. The electric potential was then calculated at the nodes located at the vertices of the elements.

1) Quasi-Stationarity: In accordance with previous studies, the EMG models were assumed to satisfy the conditions for quasistationarity [20]. This requires both that wave propagation, inductive and capacitive effects may be ignored and that the medium in which the source is located be purely resistive [3], [21]. Furthermore, if a piecewise homogeneity of the model is assumed, then the potential distribution may be determined from Laplace's equation

$$
\begin{aligned}
& \Delta \phi(r, \theta, z)=\frac{\delta^{2} \phi(r, \theta, z)}{\delta r^{2}}+\frac{1}{r} \frac{\delta \phi(r, \theta, z)}{\delta r} \\
&+\frac{1}{r^{2}} \frac{\delta^{2} \phi(r, \theta, z)}{\delta \theta^{2}}+\frac{\delta^{2} \phi(r, \theta, z)}{\delta z^{2}}=0
\end{aligned}
$$

where $\phi(r, \theta, z)$ is the potential at a point in the volume conductor of radial position $r$, angular displacement $\theta$, and axial position $z$ (Fig. 1).

2) Boundary Conditions: At the interface between any two media, the normal component of the electric current is assumed to be continuous and the following condition is satisfied

$$
\sigma_{1} E_{1 n}=\sigma_{2} E_{2 n}
$$

where $\sigma_{1}$ and $\sigma_{2}$ are the conductivities of the first and second media, respectively, and $E_{1 n}$ and $E_{2 n}$ are the normal components of the electric field in the first and second media. At the skin surface, it was assumed that the conductivity of the surrounding region (air) is zero. As current is not confined within a section of the limb, but is free to flow into the distal and proximal ends, the volume conductor was assumed to be of infinite length. This was implemented by applying boundary conditions based on the Bayliss-Turkel approximation [22] at either end of the finite-element model.

3) Source Excitation: The transmembrane current-density, $I_{m}(z)$, was applied to the surface of a muscle fiber, radius 50 $\mu \mathrm{m}$ [23], located within the conducting muscle tissue. $I_{m}(z)$ was defined as a continuous function of axial distance, $z$, which is discretised at the nodes along the surface of the fiber. The

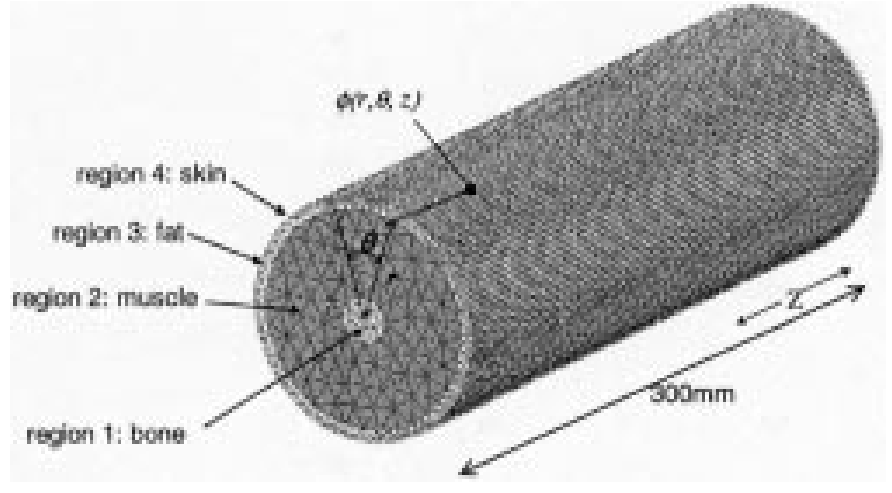

Fig. 1. Finite-element model of the upper arm incorporating muscle, fat, skin and bone (model V).

source was located centrally along the muscle length and orientated parallel to the skin surface. The current-density distribution was calculated from Rosenfalck's algebraic description of the transmembrane potential $V_{m}(z)$ [24], using the assumption that $I_{m}(z)$ is directly proportional to the second derivative of the transmembrane voltage [1], [24], [25]

$$
I_{m}(z)=\frac{\sigma_{i} \pi d^{2}}{4} \cdot \frac{d^{2} V_{m}(z)}{d z^{2}}
$$

where $\sigma_{i}$ is the intracellular conductivity and $d$ is the fiber diameter.

4) Material Properties: A parametric model to describe the dielectric properties of a range of biological tissues as a function of frequency was presented by Gabriel et al., [16]. Conductivity values for muscle $\sigma_{z}$, skin $\sigma_{s}$, infiltrated fat $\sigma_{f}$, cortical bone $\sigma_{\text {cort }}$, and cancellous bone $\sigma_{\text {canc }}$ at $100 \mathrm{~Hz}$ (representative of the median frequency of a typical surface EMG signal) were obtained from [16], Table I. A wide range of muscle anisotropy values has been reported in the literature, ranging from 2.78 to 14.5 [4], [14], [25], [26]. To examine the effect of anisotropy in the inhomogeneous model, the ratio of the axial conductivity $\sigma_{z}$ to the radial conductivity $\sigma_{r}$ of the muscle tissue was varied between one and 15. An anisotropy ratio, $\lambda$, of five was assumed elsewhere [26]-[28].

5) Implementation Details: Flat, quadrilateral elements were used to cover the skin surface with a uniform, high-resolution mesh. Three-dimensional tetrahedral elements were then used to mesh the interior regions of the model, Fig. 1. The size of the elements in the models ranged from $0.05 \mathrm{~mm}$ in the muscle fiber to an element size of $12 \mathrm{~mm}$. The total number of elements ranged from approximately 300000 to 600000 , depending on the complexity of the muscle geometry. The potential at each electrode was assumed to be the potential at the node located at the center of the electrode. The finite-element model was meshed and solved using the EMAS software package (Ansoft Corp., Pittsburgh, PA) [29], on a Dell Dimension 8100 with a Pentium IV 1.4-GHz processor, with 512-MB RAM. Typical solution time for a model containing 400000 elements was approximately $12 \mathrm{~min}$.

\section{B. Model Simulations}

For an action potential propagating with uniform conduction velocity, $u$, in a straight line, parallel to the surface of the volume 
TABLE I

MODEL INPUT PARAMETERS

\begin{tabular}{lll}
\hline \multicolumn{1}{c}{ Parameter } & \multicolumn{1}{c}{ Value } & Reference \\
\hline model length & $300 \mathrm{~mm}$ & \\
model I-V: limb radius & $50 \mathrm{~mm}$ & \\
$\begin{array}{l}\text { upper arm model: } \\
\quad \text { fat thickness } \\
\quad \text { skin thickness }\end{array}$ & $0-18 \mathrm{~mm}$ & {$[32]$} \\
$\quad$ limb radius & $1.3 \mathrm{~mm}$ & {$[33]$} \\
muscle fiber conduction velocity & $41.3-61.3 \mathrm{~mm}$ & \\
muscle conductivity (transverse & $.2455 \mathrm{~S} / \mathrm{m}$ & {$[34]$} \\
direction), $\sigma_{t}$ & & {$[14]$} \\
skin conductivity, $\sigma_{s}$ & $4.55 \times 10^{-4} \mathrm{~S} / \mathrm{m}$ & {$[14]$} \\
fat conductivity, $\sigma_{f}$ & $0.0379 \mathrm{~S} / \mathrm{m}$ & {$[14]$} \\
cortical bone conductivity, $\sigma_{c o r t}$ & $0.02 \mathrm{~S} / \mathrm{m}$ & {$[14]$} \\
cancellous bone conductivity, & $0.075 \mathrm{~S} / \mathrm{m}$ & {$[14]$} \\
$\begin{array}{l}\sigma_{c a n c} \\
\text { intracellular conductivity, } \sigma_{i}\end{array}$ & $1.01 \mathrm{~S} / \mathrm{m}$ & {$[23]$} \\
anisotropy ratio, $\lambda$ & 5 & {$[25]$} \\
(physiological model) & & {$[24]$} \\
muscle fiber diameter, $d$ & $50 \mu \mathrm{m}$ & {$[23]$} \\
transmembrane voltage, $V_{m}(z)$ & $V_{m}(z)=96 z^{3} \cdot e^{-z}-90$ & \\
source length & $15 \mathrm{~mm}$ & \\
\hline \hline
\end{tabular}

conductor, potential can be viewed as a function of either axial position, $z$, or time, $t$, according to the relationship $z=u t$. Additional to the propagating wave, the EMG signal detected at the skin surface contains a stationary component, generated as the action potential reaches the end of the fiber [3], [30]-[32]. The contribution of the fiber end-effects at the recording electrode depends on the electrode position relative to the fiber terminal, the distance from fiber to electrode and the interelectrode distance. During bipolar recordings, where the electrodes are situated away from the end of the fiber, the stationary waveform tends to cancel out [5]. Furthermore, due to the dispersion of the fiber terminations within a motor unit, the end-effects observed in a motor unit action potential are much smaller than those observed for a single fiber action potential. For this reason, muscle fiber end-effects are not included in this study and it is assumed that the EMG signal is dominated by the propagating wave. The effect of the action potential origination at the neuromuscular junction is much less dramatic than the termination effects at the end of the fiber, as the effects due to two action potentials propagating in opposite directions to one another tend to cancel out. Action potential origination effects are, therefore, not considered here. However, if the recording electrodes are located close to the innervation zone the start-up effects could be a significant factor.

Five variations on a model of the upper arm are initially presented. In the first, the source was embedded in cylindrical, homogeneous anisotropic muscle tissue of finite radial extent (Table II: model I). In the second and third models, the outer rim of the volume conductor was replaced with a layer of isotropic skin (Table II: model II), and then isotropic fat (Table II: model III), 2-mm-thick and 5-mm-thick, respectively. A fourth model was composed of 2-mm-thick skin, 3-mm-thick fat and muscle (Table II: model IV). A cylindrical bone, radius $10 \mathrm{~mm}$ [33] was added to a fifth model (Table II: model V). Using model V, the
TABLE II

TISSUE COMPOSITION OF MODELS I-V

\begin{tabular}{llllll}
\hline \hline & Model I & Model II & Model III & Model IV & Model V \\
\hline Region 1 & muscle & muscle & muscle & muscle & bone \\
Region 2 & muscle & muscle & muscle & muscle & muscle \\
Region 3 & muscle & muscle & fat & fat & fat \\
Region 4 & muscle & skin & fat & skin & skin \\
\hline \hline
\end{tabular}

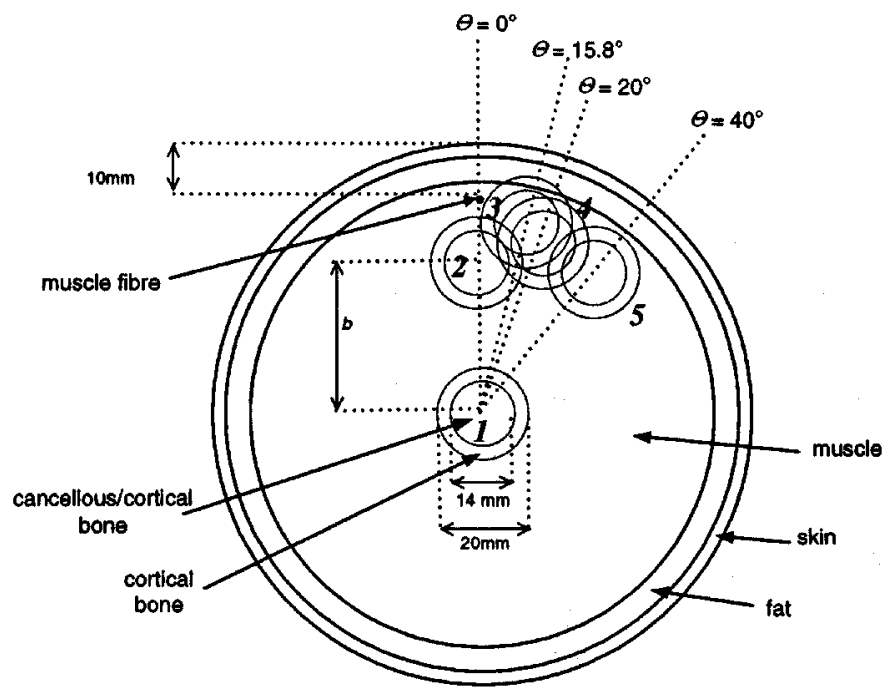

Fig. 2. Cross section of model illustrating simulated bone locations. $b$ denotes the distance from center of the volume conductor to the center of bone, $\Theta$, the angular displacement of the bone from the source.

location of the bone with respect to the muscle fiber was varied as illustrated in Fig. 2 ( $b$ denotes the distance from center of the volume conductor to the center of bone and $\Theta$, the angular displacement of the bone from the vertical axis). The bone was first located at the center of the model, then $2 \mathrm{~mm}$ below the source. The bone was then rotated $15.8^{\circ}$ from the vertical and moved so that the edge of the bone lay $1 \mathrm{~mm}$ below the fat-skin interface and $1 \mathrm{~mm}$ from the source. Finally, the bone was placed $2 \mathrm{~mm}$ below the muscle-fat interface at angular displacements of $20^{\circ}$ and $40^{\circ}$ from the source. Two types of bone were compared at each location. Initially, the bone was modeled as homogeneous cortical bone tissue. Then the center of the bone, radius $7 \mathrm{~mm}$, was filled with less resistive cancellous tissue. In models I-V, the total radius of the volume conductor was $50 \mathrm{~mm}$. The amplitude and frequency content of the surface EMG signals from the different models were compared and the rate of decay of the surface EMG root-mean-square (RMS) value was examined.

To explore the effect of variations in subcutaneous tissue thickness, surface EMG action potentials were then generated using a multilayer model of the upper arm as the thickness of the subcutaneous fat was varied while keeping the volume of the muscle tissue constant. This model was composed of skin, fat, muscle and cortical bone, radius $10 \mathrm{~mm}$, located at the center of the model. The radius from the center of the model to the surface of the muscle tissue was $40 \mathrm{~mm}$. It has been reported that biceps skin-fold thickness ranges from $2-29 \mathrm{~mm}$ in males, and from 2-45 mm in females, aged 20-40 years [34]. Based on this data and assuming a skin thickness of $1.3 \mathrm{~mm}$ [35], the 


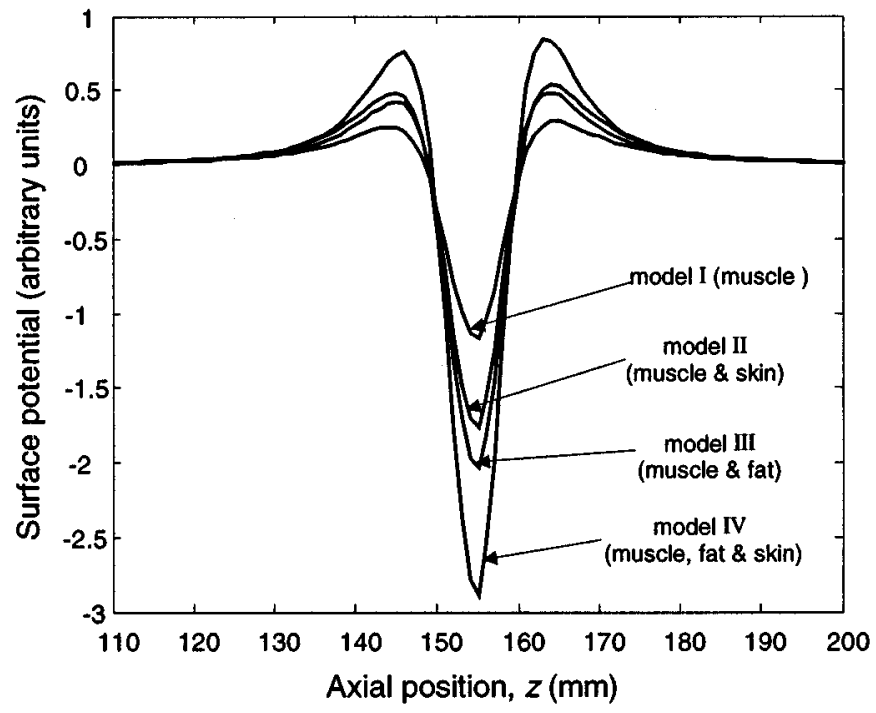

Fig. 3. Comparison of action potentials detected at the surface directly above a fiber of depth of $7 \mathrm{~mm}$ in models I-IV.

thickness of the subcutaneous fat layer was varied between 0 and $18 \mathrm{~mm}$ and the amplitude, frequency content and rate of decay of the surface EMG signal with angular displacement from the source was examined.

\section{Simulation RESUlTS}

Action potentials were examined at different angular locations $\theta$ around the surface of the volume conductor. The median frequency of the EMG amplitude spectrum (magnitude of the Fourier transform) was calculated by mapping the spatially distributed waveform to the time domain, assuming a conduction velocity of $4 \mathrm{~m} / \mathrm{s}$ [36]. Results are presented for a standard bipolar electrode configuration, with an interelectrode distance of $20 \mathrm{~mm}$, orientated parallel to the muscle fiber direction.

\section{A. Effect of Inhomogeneous Layers}

Action potentials detected at the surface, directly above a 7-mm-deep muscle fiber in models I-IV are compared in Fig. 3. Replacing the outer layer of the homogeneous model (model I) with a medium of low conductivity, such as skin or fat tissue, yielded an increase in both the amplitude and frequency content of the surface action potential. The RMS value and median frequency for a range of fiber depths are compared in Fig. 4(a) and (b). Examining the potential directly above a 7-mm-deep muscle fiber at the muscle-fat interface in models I and III, it was observed that the RMS value of the action potential at the interface increased by $90 \%$ when the outer layer of the volume conductor was replaced with fat tissue. This was accompanied by a decrease in the total current entering the outer annulus.

When the conductivity of the outer layer of the volume conductor was decreased further (beyond physiologically realistic values), the amplitude and median frequency of the surface action potential continued to increase, approaching a limit as the conductivity of the outer layer was decreased toward zero. In Fig. 5, the RMS value and median frequency of the surface potential directly above the source (at $\theta=0^{\circ}$ ) are presented as a function of the ratio of muscle conductivity to fat conductivity

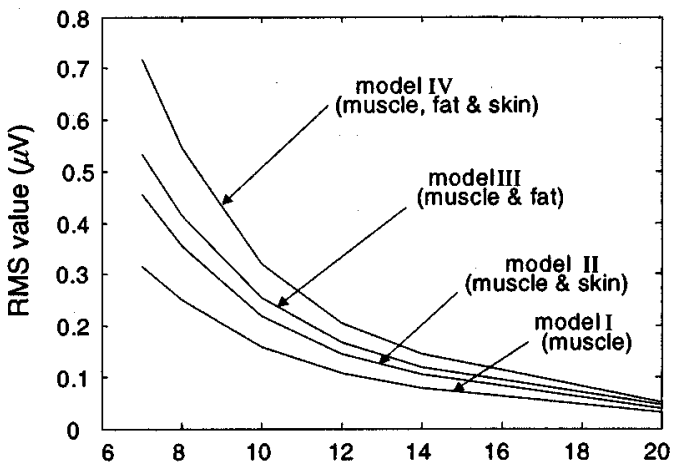

(a)

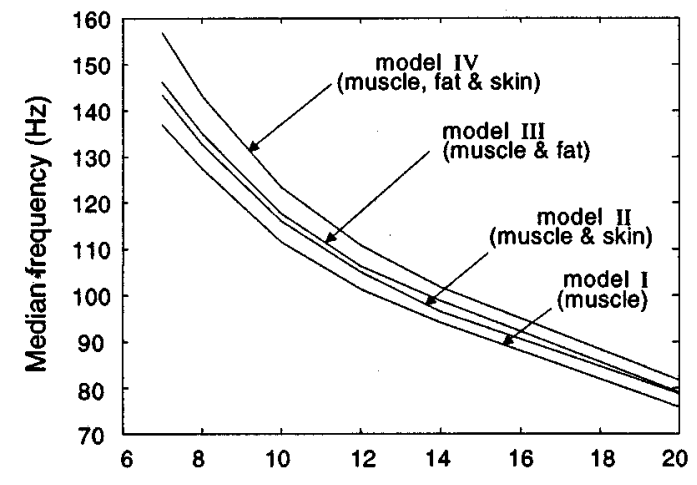

(b)

Fig. 4. (a) RMS value and (b) amplitude spectrum median frequency of surface potentials directly above the active fiber as fiber depth (in millimeters) is varied.

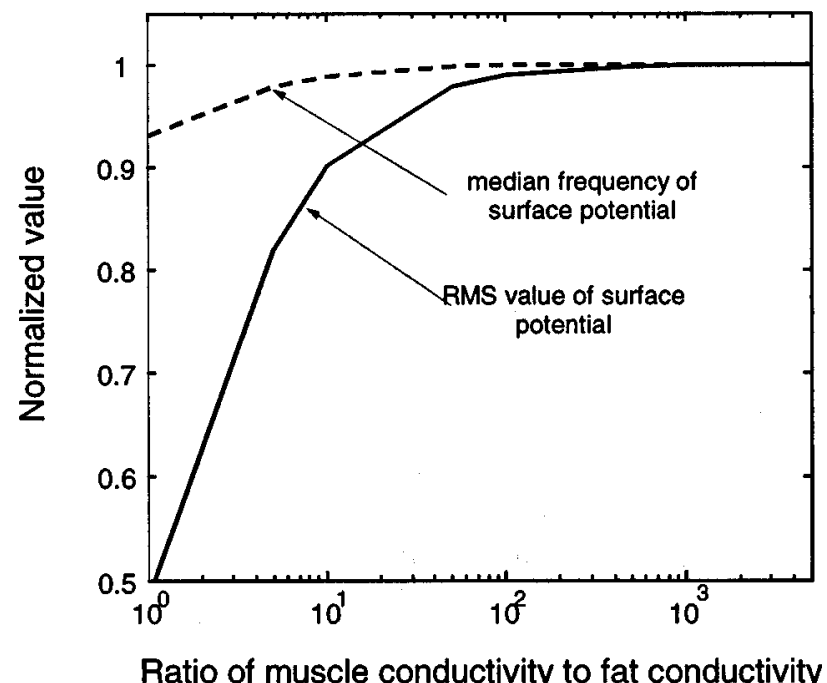

Fig. 5. Normalized RMS value (solid line) and amplitude spectrum median frequency (dashed line) of surface potentials detected directly above a 7-mm-deep muscle fiber in model III, as the ratio of the muscle conductivity to fat conductivity is varied.

in model III. Both variables have been normalized with respect to their maximum values.

When highly resistive fat or skin was included in the model, the rate of decay of the surface EMG amplitude with increasing source depth was observed to increase, Fig. 4(a). The rate of decay of the surface EMG amplitude with increasing angular displacement from the source is illustrated in Fig. 6. Results are presented for a muscle fiber located $7 \mathrm{~mm}$ below the surface 


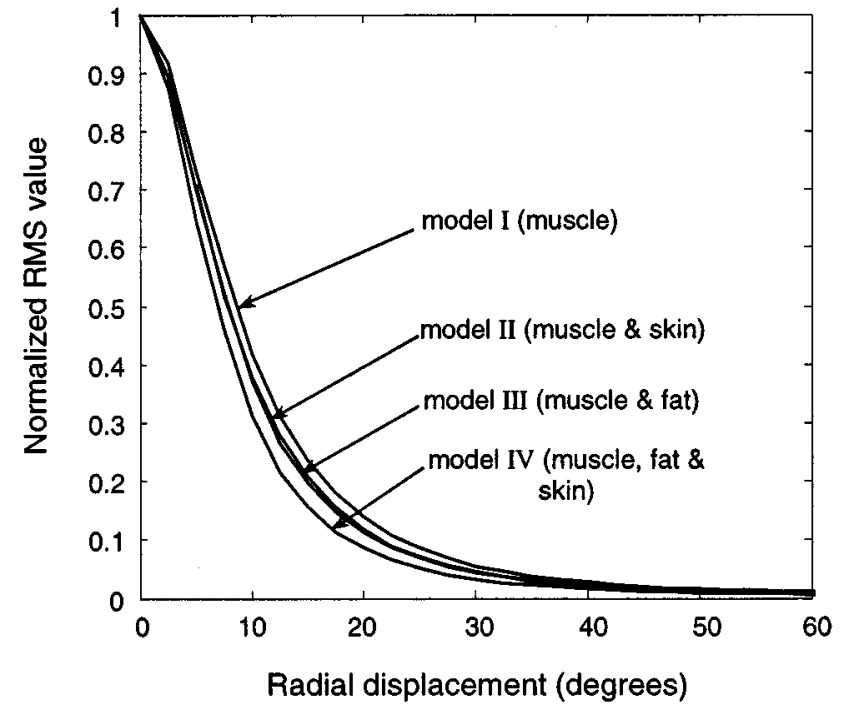

Fig. 6. Rate of decay of the surface EMG RMS amplitude with increasing angular displacement from a 7-mm-deep muscle fiber.

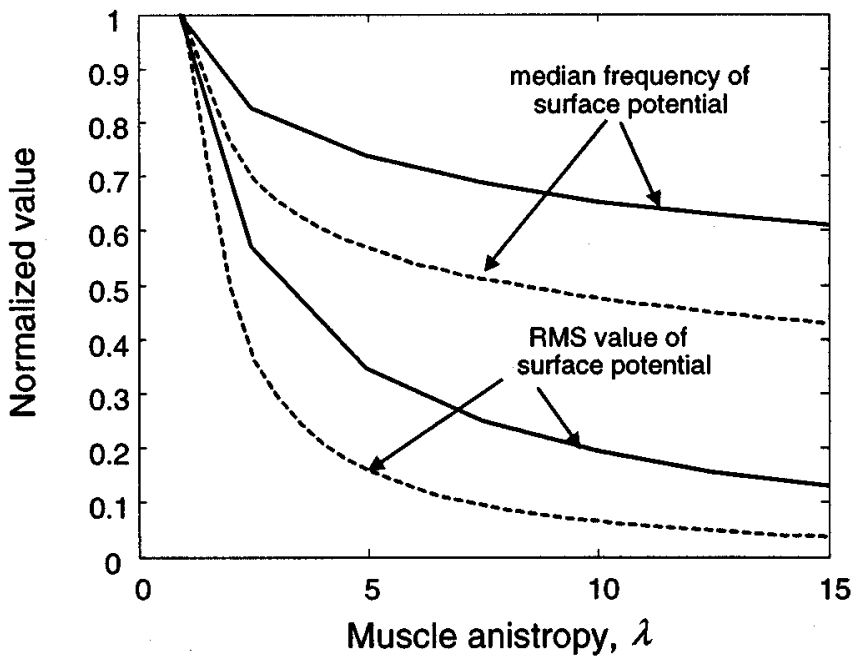

Fig. 7. RMS value and median frequency of surface potentials detected directly above a 7-mm-deep muscle fiber, as muscle anisotropy, $\lambda$, is varied in model I (solid line) and model IV (dashed line). Variables are normalized with respect to their maximum values.

of the model. The results illustrate that in models where highly resistive layers were included (models II-IV), the surface potential decayed more rapidly with angular displacement than in the homogeneous muscle model.

\section{B. Effect of Muscle Anisotropy}

Muscle anisotropy was increased by increasing the axial conductivity of the muscle tissue in the homogeneous muscle model (model I) and in the multilayer skin, fat and muscle model (model IV). The RMS value and median frequency of the action potential detected at the surface directly above a 7-mm-deep muscle fiber in both models are presented in Fig. 7. The decrease in both the amplitude and frequency content of the surface potentials with increasing muscle anisotropy is characteristic of the changes that were observed at all electrode locations around the limb. Maintaining the muscle fiber at

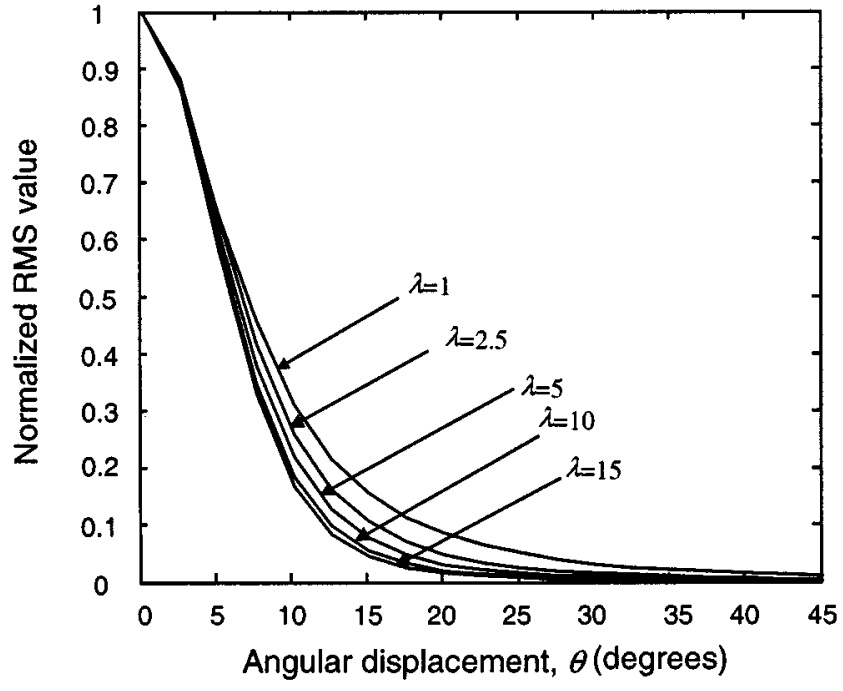

Fig. 8. Rate of decay of surface potential RMS value with increasing angular displacement from the source, as muscle anisotropy is varied.

a depth of $7 \mathrm{~mm}$, the rate of decay of the surface potential around the limb with increasing angular displacement from the source was examined as $\lambda$ was varied. The RMS value of the surface potential was calculated at $2.5^{\circ}$ intervals around the volume conductor and normalized with respect to the RMS value at $\theta=0^{\circ}$. The rate of decay of the surface potential with increasing angular displacement was observed to increase slightly with increasing $\lambda$. Values for the inhomogeneous model (model IV) are presented in Fig. 8.

\section{Effect of Bone}

The RMS values of action potentials detected at $2.5^{\circ}$ intervals around the surface of the limb were calculated for each bone location illustrated in Fig. 2 using both homogeneous cortical bone and the two-layer bone composed of an outer layer of cortical bone and a core of cancellous bone. In Fig. 9, the increase in surface potential RMS value with respect to the peak value calculated in the absence of bone is presented at each angular location, $\theta$. Results for both the homogeneous cortical bone and the cortical and cancellous bone at each of the five locations are presented. Placing the bone at the center of the model (position 1) had a relatively small effect on the potentials detected at the skin surface, with no discernable difference between the cortical and mixed-tissue bone. As the bone was moved closer to the source (position 2), a substantial increase in the amplitude of the surface EMG signal above the bone was observed, and a slight difference between the two types of bone was detected. Similarly, when the bone was placed close to the muscle fat interface, next to the source (positions 3-5) the RMS value of the surface potentials increased between the source and bone, and directly above the bone, while on the opposite side of the bone a slight decrease in the amplitude of the surface potential was observed, Fig. 9.

\section{Effect of Increasing Subcutaneous Fat Thickness}

To investigate how variations in fat thickness among subjects and over different muscles affect the surface EMG signal, the potential at the skin surface was examined as the thickness of 


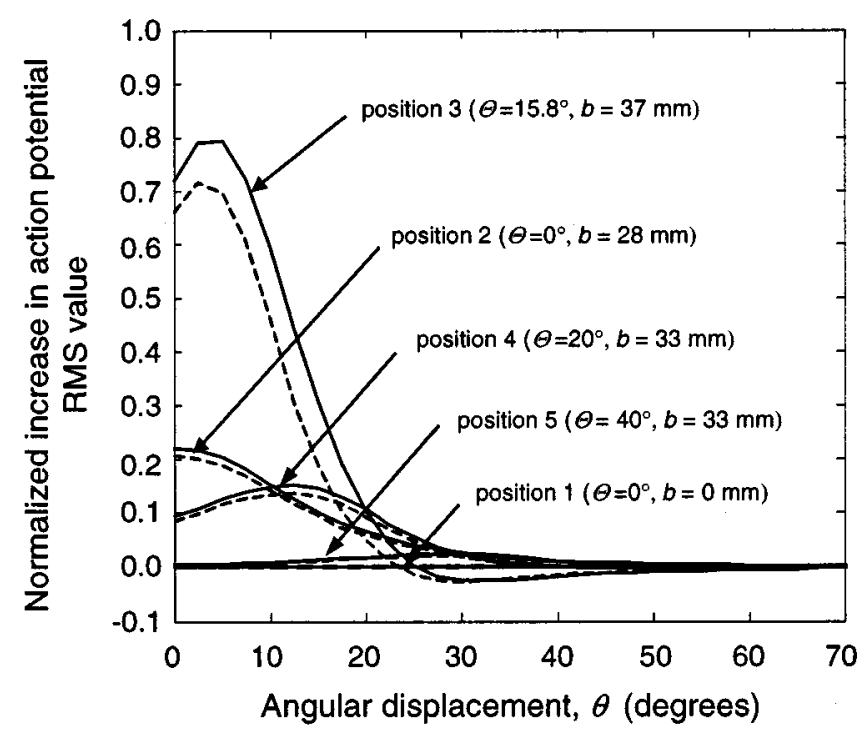

Fig. 9. Change in surface potential RMS value with the addition of bone at locations 1-5. Values for the cortical bone are indicated with a solid line, values for the cortical bone with a core of cancellous tissue are indicated with a dashed line. Values are normalized with respect to the peak value at $\theta=0^{\circ}$ without bone. Muscle fiber depth is $10 \mathrm{~mm}$.

the subcutaneous fat layer was varied, while keeping the position of the source with respect to the muscle-fat interface and the volume of the muscle tissue constant. The RMS value and median frequency of a surface action potential observed directly above a source located $3 \mathrm{~mm}$ below the muscle-fat interface are presented in Fig. 10, as the thickness of the subcutaneous fat was increased from $0 \mathrm{~mm}$ to $18 \mathrm{~mm}$ while keeping the location of the fiber within the muscle tissue constant. At all source depths, increasing the thickness of the subcutaneous fat resulted in a decrease in the amplitude and frequency content of the surface EMG signal at all points around the limb. However, the reduction in both amplitude and frequency content was substantially lower than in a homogeneous muscle model for the same distance between electrode and source, Fig. 10.

As the thickness of the fat layer was increased, a simultaneous decrease in the rate of decay of the surface EMG signal around the limb was also noted. In Fig. 11, the rate of decay of the action potential RMS amplitude is presented as the thickness of the subcutaneous fat layer (im millimeters) was varied, for a source located $3 \mathrm{~mm}$ below the surface of the muscle-fat tissue interface.

\section{DISCUSSION}

A finite-element model of the surface EMG signal has been presented. Important features of the model include the finite nature of the conducting volume, anisotropic muscle tissue, isotropic skin and subcutaneous fat tissue, bone of arbitrary location and realistic muscle fiber transmembrane potentials. Neural control is not considered, rather the focus has been on understanding how multiple layers of inhomogeneous tissue and large inhomogeneities such as bone affect the single fiber action potentials which form the building blocks of the complex surface EMG signal. In particular, the influence of skin, subcutaneous tissue and bone on the rate of decay of the EMG

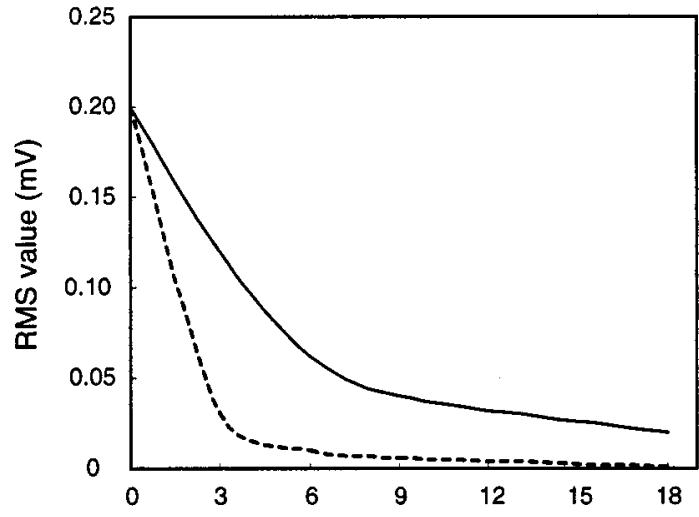

(a)

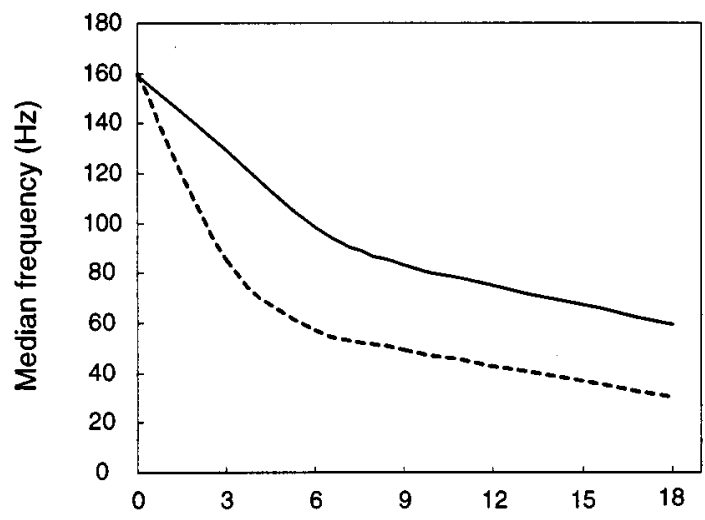

(b)

Fig. 10. (a) RMS value and (b) median frequency of an action potential detected directly above a muscle fiber located $3 \mathrm{~mm}$ below the muscle-fat interface as the thickness of the fat tissue is increased (solid line). The RMS value and median frequency for the same fiber and electrode locations in a homogeneous muscle model are also presented (dashed line).

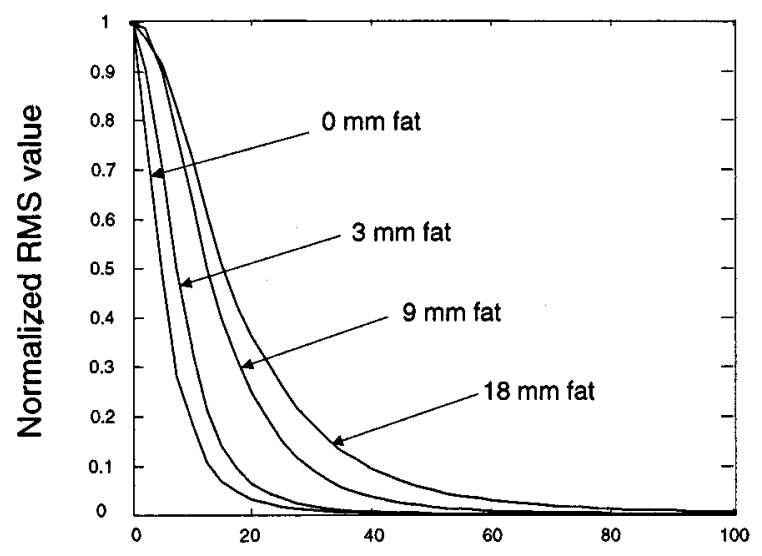

Fig. 11. Rate of decay of surface potential RMS value with increasing angular displacement from the source for a fiber located $3 \mathrm{~mm}$ below the surface of the muscle tissue, as subcutaneous fat tissue thickness is varied.

signal around the surface of the limb has been considered, as this has important implications for estimating surface EMG crosstalk. The sensitivity of the surface electrode pick-up range to electrode configuration and interelectrode distance has been discussed in detail elsewhere, both experimentally [37]-[39] and in model simulation [40]-[42]. This topic is, therefore, not addressed here and the results have been given for a standard 
single differential electrode configuration with an interelectrode distance of $20 \mathrm{~mm}$. Electrodes were modeled as point electrodes so that the potential could be examined at all points on the mesh at the surface of the model. Previous studies have reported only minor modifications in the detected waveform as electrode size is varied [40], [43]. For very superficial muscle fibers, EMG amplitude tends to decrease with increasing electrode size, however, these differences disappear as the distance between the electrode and muscle fiber is increased.

Replacing the outer layer of the homogenous muscle model with a highly resistive material such as fat or skin resulted in an increase in the amplitude and frequency content of the potential observed at the surface of the volume conductor, Figs. 3-5. These perhaps counter-intuitive results are explained by the dramatic increase in the potential at the muscle-fat interface as the current entering the outer annulus decreases, due to the increased resistance of the fat tissue. As the conductivity of the fat is decreased further, the potential at the muscle-fat interface and, hence, at the surface of the volume conductor, continues to increase as progressively more current is confined within the muscle tissue. Although the voltage across the fat tissue also increases with its resistivity, the rise in potential at the interface is greater, yielding a net increase at the surface of the model. At very low values of fat conductivity (below the range of biological tissue) the potential at the surface remains essentially constant as almost all of the current is constrained within the muscle tissue, Fig. 5. The closer the muscle fiber to the boundary of the muscle tissue, the more dramatic the voltage increase in this region. As a result, the rate of decay of the surface EMG signal, both with increasing source depth and with increasing angular displacement from the source, increases when the outer annulus of the model is filled with a medium of low conductivity such as fat or skin, Figs. 4(a) and 6. The increase in amplitude and more rapid decay with source depth observed are in agreement with observations made by Roeleveld et al. [5] using an analytical multilayer EMG model. However, in [5] a value of skin conductivity considerably higher than the measured skin conductivities reported in [14] was used. This tends to reverse the effect of the highly resistive subcutaneous tissue, rather than accentuate it as in the model presented here.

In reality, skin is a complex, laminar structure. Skin impedance consists mainly of the impedance of the stratum corneum [44], while the dermis forming the bulk of the skin thickness $(0.5-2 \mathrm{~mm})$ is significantly more hydrated and has a rich network of structures, making it much more conductive. In the model presented here, skin is modeled as a homogeneous structure using the conductivity for composite skin measured by Gabriel et al. [15] in vivo. The dielectric properties measured represent values for composite skin and lie between the values for the highly resistive stratum corneum and the more conductive tissue of the dermis [15]. It is expected that when the distance between the fiber and the electrode and the interelectrode distance are large relative to the skin thickness, homogeneous skin would yield similar results to a laminar structure.

As previously reported [4], [25], [42], increasing muscle anisotropy by increasing the muscle conductivity along the longitudinal axis, results in a reduction in the amplitude and frequency content of the simulated action potential, Fig. 7 . In the inhomogeneous model, these changes are slightly less pronounced due to the isotropic nature of the skin and adipose tissue. Increasing $\lambda$ also caused the EMG signal to decay more rapidly around the surface of the volume conductor, which would result in lower levels of EMG crosstalk, Fig. 8.

The effect of a highly resistive region of bone on the surface potential varied considerably, depending primarily on the location of the bone with respect to the source and the boundary of the volume conductor. When located at the center of the model, the bone had little effect on simulated surface potentials and no difference between the homogenous cortical bone and the bone with a core of cancellous tissue was detected, Fig. 9. However, as the bone was moved closer to the source, an increase in surface EMG amplitude was observed between the source and the bone, and directly above the bone, where current flow was constrained. On the opposite side of the bone, where current flow had been subsequently blocked, a slight decrease in the surface potential was observed. These effects can have a marked impact on surface EMG crosstalk, causing it to increase above the bone and to one side of it, while reducing on the opposite side. Although the effect of bone would appear to be minor in the upper arm since the humerus is located approximately at the center of the limb, in areas where the bone is located close to active fibers and to the surface of the volume conductor, it is clear that its influence on the surface EMG signal can be significant.

Replacing regions of a homogeneous model with tissues of varying conductivity provides insight into the conduction of the EMG signal through an inhomogeneous volume conductor. In experimental applications, however, it is more likely that variations in fat thickness among subjects and over different muscles are observed as the location of the fibers within the muscle tissue remains relatively constant. Changes in the surface EMG signal were, therefore, examined as the thickness of the fat layer was increased while keeping the location of the fiber with respect to the muscle-fat interface and the volume of the muscle fixed. As the thickness of the fat layer and, hence, the distance between fiber and electrode increased, the amplitude and frequency content of the surface potential decreased, Fig. 10. Spatial filtering characteristics alone predict that surface EMG amplitude and frequency content will decrease as the distance between the electrode and the fiber increases. However, the amplitude and frequency decreases observed in the multilayer model as fat thickness increased were substantially less than those observed in the homogeneous muscle model, Fig. 10. When the region between the fiber and the surface of the model is partly filled with highly resistive fat tissue, the amplitude and frequency content of the EMG signal at the surface are higher than in the homogeneous model for a fixed electrode-fiber distance, Fig. 4. Consequently, the spatial filtering of the EMG signal due to increased fiber-electrode distance predicted by a homogeneous muscle model is markedly reduced when the increased distance is due to a thickening of a more resistive fatty tissue, Fig. 10.

In an extensive study of surface and intramuscular crosstalk in the muscles of the cat hind limb, Solomonow et al. [17] reported a dramatic increase in surface EMG crosstalk in muscles covered by substantial amounts of subcutaneous fat. Similarly, 
it was illustrated in [18] that the selectivity of surface EMG signals from the biceps muscle decreases as skinfold thickness increases. The reasons behind these observations are not immediately obvious. Using the finite-element model, the relationship between subcutaneous tissue thickness and the rate of decay of the surface action potential around the limb was examined. The simulated results demonstrate a decrease in the rate of decay of the surface potential with angular displacement as subcutaneous tissue thickness increased and, hence, an increase in surface EMG crosstalk, consistent with the experimental observations, Fig. 11. As illustrated in Fig. 6, replacing regions of the model with fat and skin tissue caused the amplitude at the surface to decay more rapidly. It is, therefore, suggested that the correlation between skin-fold thickness and crosstalk observed experimentally is due to the increased distance between the active fibers and recording electrodes, rather than the material properties of the adipose tissue. As the subcutaneous tissue thickness increases, the relative variation in distance between the active fibers and the electrode at each point around the limb reduces, yielding more similar EMG signals at each detection point.

In accordance with previous studies, it has been assumed here that biological tissues behave as if they are purely resistive at the frequencies of interest. The conductivities and permitivities of muscle and fat described by the parametric models of Gabriel et al. [16] satisfy the requirements of a resistive model outlined in [20]. However, a wide range of values has been reported in the literature and other combinations of conductivity and permittivity may yield more significant capacitive effects [45]. Similarly, at distances very close to the active fiber, studies using a microscopic network model [17], [46] have indicated that it is necessary to incorporate membrane capacitance and the frequency-dependent nature of the tissue. Although the values reported in [14] suggest that large capacitive currents may exist in skin tissue, skin thickness is in the range of only 1-2 mm [35]. The influence of skin capacitance is, therefore, more difficult to predict and requires further exploration.

\section{CONCLUSION}

Finite-element analysis has been shown to be a useful tool for examining the effect of material properties on simulated EMG signals in volume conductors of arbitrary geometry. The inclusion of highly resistive layers of fat and skin tissue causes an increase in the amplitude and frequency content of the simulated surface EMG signal, when compared with a homogeneous muscle model for the same electrode and muscle fiber location. This is due to the increased electrical potential at the interface of the muscle and fat tissues as more current becomes constrained within the muscle tissue. The rate of decay of the surface potential with increasing source depth and with increasing angular displacement from the source is also observed to increase. Subcutaneous tissue, however, is generally associated with an attenuation and low-pass filtering of the surface EMG signal. Based on the simulation results, it is concluded that the attenuation and reduced frequency content of the surface EMG signal observed as subcutaneous tissue thickness increases is due to the increased distance between the muscle fibers and the recording site, rather than the relatively high resistance of the subcutaneous tissue. The spatial filtering of the signal as subcutaneous fat thickness increases is significantly lower than that predicted by a homogeneous muscle model. It is important, therefore, to distinguish between the effect of material properties and distance between source and electrodes when considering the effect of subcutaneous fat. The results also indicate that the effect of bone, which is seldom considered in either experimental or simulation EMG studies, is not always negligible. In regions where the bone lies close to the active muscle fibers and the skin surface, the distribution of the electrical potential at the skin surface may be significantly altered.

\section{REFERENCES}

[1] R. Plonsey, "The active fiber in a volume conductor," IEEE Trans. Biomed. Eng., vol. BME-21, pp. 371-381, 1974.

[2] J. Clark and R. Plonsey, "The extracellular potential field of the single active nerve fiber in a volume conductor," Biophys. J., vol. 8, pp. 842-864, 1968.

[3] T. H. J. M. Gootzen, D. F. Stegeman, and A. Van Oosterom, "Finite dimensions and finite muscle length in a model for the generation of electromyographic signals," Electroencephalogr. Clin. Neurophysiol., vol. 81, pp. 152-162, 1991.

[4] N. Ganapathy, J. W. Clark, and O. B. Wilson, "Extracellular potentials from skeletal muscle," Math. Biosci., vol. 83, pp. 61-96, 1987.

[5] K. Roeleveld, J. H. Blok, D. F. Stegeman, and A. Van Oosterom, "Volume conduction models for surface EMG; Confrontation with measurements," J. Electromyogr. Kinesiol., vol. 7, pp. 221-232, 1997.

[6] B. K. Van Veen, N. J. M. Rijkhoff, W. L. C. Rutten, W. Wallinga, and H. B. K. Booom, "Potential distribution and single action potentials in a radially bounded muscle model," Med. Biol. Eng. Comput., vol. 30, pp. 303-310, 1992.

[7] A. Heringa, D. F. Stegeman, G. J. H. Uijen, and J. P. C. De Weerd, "Solution methods of electrical field problems in physiology," IEEE Trans. Biomed. Eng., vol. BME-29, pp. 34-42, 1981.

[8] J. Schneider, S. Silny, and G. Rau, "Influence of tissue inhomogeneties on noninvasive muscle fiber conduction velocity measurements-Investigated by physical and numerical modeling," IEEE Trans. Biomed. Eng., vol. 38, pp. 851-860, Sept. 1991.

[9] A. T. Barker, B. H. Brown, and I. L. Freeston, "Modeling of an active nerve fiber in a finite volume conductor and its application to the calculation of surface action potentials," IEEE Trans. Biomed. Eng., vol. BME-26, pp. 53-56, 1979.

[10] S. M. Wood, J. A. Jarratt, A. T. Barker, and B. H. Brown, "Surface electromyography using electrode arrays: A study of motor neuron disease," Muscle Nerve, vol. 24, pp. 223-230, 2001.

[11] N. A. Trayanova, "Electrical behavior of a skeletal muscle fiber in a volume conductor of finite extent," Biol. Cybern., vol. 63, pp. 121-125, 1990.

[12] D. Farina and A. Rainoldi, "Compensation of the effect of sub-cutaneous tissue layers on surface EMG: A simulation study," Med. Eng. Phys., vol. 21, pp. 487-496, 1999.

[13] O. B. Wilson, J. W. Clark, N. Ganapathy, and T. L. Harman, "Potential field from an active nerve in an inhomogeneous, anisotropic volume conductor-The forward problem," IEEE Trans. Biomed. Eng., vol. BME-32, pp. 1032-1041, 1985.

[14] C. Gabriel, S. Gabriel, and E. Corthout, "The dielectric properties of biological tissues: I literature survey,” Phys. Med. Biol., vol. 41, pp. 2231-2249, 1996

[15] S. Gabriel, R. W. Lau, and C. Gabriel, "The dielectric properties of biological tissues: II measurements in the frequency range $10 \mathrm{~Hz}$ to 20 GHz,” Phys. Med. Biol., vol. 41, pp. 2251-2269, 1996.

[16] — - "The dielectric properties of biological tissues: III parametric models for the dielectric spectrum of tissues," Phys. Med. Biol., vol. 41, pp. 2271-2293, 1996.

[17] M. Solomonow, R. Baratta, M. Bernadi, B. Zhou, Y. Lu, M. Zhu, and S. Acierno, "Surface and wire EMG cross-talk in neighboring muscles," $J$. Electromyogr. Kinesiol., vol. 4, pp. 131-142, 1994.

[18] E. J. De la Barrera and T. M. Milner, "The effect of skinfold thickness on the selectivity of surface EMG," Electroencephalogr. Clin. Neurophysiol., vol. 93, pp. 91-99, 1994. 
[19] C. J. De Luca and R. Merletti, "Surface myoelectric cross-talk among muscles of the leg," Electroencephalogr. Clin. Neurophysiol., vol. 70, pp. 2010-2023, 1988

[20] R. Plonsey and D. B. Heppner, "Considerations of quasistationarity in electrophysiological systems," Bull. Math. Biophys., vol. 29, pp. $657-665,1967$.

[21] H. P. Schwan and C. F. Kay, "The conductivity of living tissues," Ann. N.Y. Acad. Sci., vol. 65, pp. 1007-1013, 1957.

[22] A. Taflove and S. Hagness, Computational Electrodynamics: The FiniteDifference Time-Domain Method, 2nd ed. Boston, MA: Artech House, 2000.

[23] V. J. Basmajian and C. J. De Luca, Muscles Alive, 5th ed. Baltimore, MD: Williams \& Wilkins, 1985.

[24] P. Rosenfalck, "Intra and extracellular potential fields of active nerve and muscle fibers," Acta Physiol. Scand., no. Suppl. 321, pp. 1-168, 1969.

[25] S. Andreassen and A. Rosenfalck, "Relationship of intracellular and extracelular action potentials of skeletal muscle fibers," CRC Crit. Rev. Bioeng., vol. 6, pp. 267-306, 1981.

[26] F. L. H. Gielen, W. Wallinga-de Jonge, and K. L. Boon, "Electrical conductivity of skeletal muscle tissue: Experimental results from different muscles in vivo," Med. Biol. Eng. Comput., vol. 22, pp. 569-577, 1984.

[27] L. A. Geddes and L. E. Baker, "The specific resistance of biological material-A compendium of data for the biomedical engineer and physiologist," Med. Biol. Eng. Comput., vol. 5, p. 271, 1967.

[28] S. D. Nandedkar and E. Stalberg, "Simulation of single muscle fiber action potentials," Med. Biol. Eng. Comput., vol. 22, pp. 564-568, 1983.

[29] B. E. Mac Neal, J. R. Brauer, and R. N. Coppolino. A general finiteelement vector potential formulation of electromagnetics using a timeintegrated scalar potential. presented at INTERMAG'90. [CD-ROM]. Available: CD-6

[30] N. Dimitrova, "Model of the extracellular potential field of a single striated muscle fiber," Electromyogr. Clin. Neurophysiol., vol. 14, pp. 53-66, 1974.

[31] A. Gydikov, L. Gerilovsky, N. Radicheva, and N. Trayanova, "Extracellular potentials of single active muscle fibers: Effect of fiber length," Biol. Cybern., vol. 54, pp. 1-8, 1986.

[32] P. Kleinpenning, T. Gootzen, A. Van Oosterom, and D. Stegeman, "The equivalent source description representing the extinction of an action potential at a muscle fiber ending," Math. Biosci., vol. 101, pp. 41-61, 1990.

[33] S. B. Heymsfield, C. Mc Manus, J. Smith, V. Stevens, and D. W. Nixon, "Anthropometric measurements of muscle mass: Revised equations for calculating bone-free arm muscle area," Amer. J. Clin. Nutr., vol. 36, pp. 680-690, 1982

[34] J. V. G. A. Durnin and J. Womersley, "Body fat assessed from total body density and its estimation from skinfold thickness: Measurements on 481 men and women aged from 16 to 72 years," Br. J. Nutr., vol. 32, pp. 77-97, 1974.

[35] W. S. Snyder, M. J. Cook, L. R. Karhausen, E. S. Nasset, G. P. Howells, and I. H. Tipton, International Commission on Radiological Protection no. 23. Oxford, U.K.: Pergamon, 1974.

[36] E. Stalberg and J. V. Tronelj, Single Fiber Electromyography, 2nd ed. New York: Raven, 1994.

[37] T. J. Koh and M. D. Grabiner, "Cross talk in surface electromyograms of human hamstring muscles," J. Orth. Res., vol. 10, pp. 701-709, 1992.

[38] G. Rau and C. Disselhorst-Klug, "Principles of high-spatial resolution surface EMG (HSR-EMG): Single motor unit detection and application in the diagnosis of neuromuscular disorders," J. Electromyogr. Kinesiol., vol. 7, pp. 233-239, 1997.

[39] J. P. P. van Vugt and J. G. van Dijk, "A convenient method to reduce crosstalk in surface EMG," Clin. Neurophysiol., vol. 112, pp. 583-592, 2001.

[40] A. J. Fuglevand, D. A. Winter, A. E. Patla, and D. Stashuk, "Detection of motor unit action potentials with surface electrodes: Influence of electrode size and spacing," Biol. Cybern., vol. 67, pp. 143-153, 1992.

[41] D. A. Winter, A. J. Fuglevand, and S. E. Archer, "Cross-talk in surface electromyography: Theoretical and practical estimates," J. Electromyogr. Kinesiol., vol. 4, pp. 15-26, 1994.

[42] R. Merletti, L. Lo Conte, E. Avignone, and P. Guglielminotti, "Modeling of surface myoelectric signals-Part I: Model implementation," IEEE Trans. Biomed. Eng., vol. 46, pp. 810-820, July 1999.

[43] P. A. Lynn, N. D. Bettles, A. D. Hughes, and S. W. Johnson, "Influences of electrode geometry on bipolar recordings of the surface electromyogram," Med. Biol. Eng. Comput., vol. 16, pp. 651-660, 1978.
[44] T. Yamamoto and Y. Yamamoto, "Dielectric constant and resistivity of epidermal stratum corneum," Med. Biol. Eng. Comput., vol. 14, pp. 494-500, 1976.

[45] N. S. Stoykov, M. Lowery, A. Taflove, and T. A. Kuiken, "A finite element analysis of muscle tissue capacitive effects and dispersion in EMG," presented at the 23rd Int. Conf. IEEE Eng. in Med. Biol., Istanbul, Turkey, 2001

[46] B. A. Albers, W. L. C. Rutten, W. Wallinga-de Jonge, and H. B. K. Boom, "A model study on the influence of structure and membrane capacitance on volume conduction in skeletal muscle tissue," IEEE Trans. Biomed. Eng., vol. BME-33, pp. 681-689, 1986.

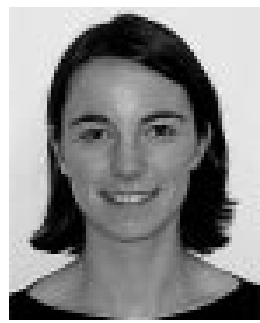

Madeleine M. Lowery (M'00) received the B.E. and Ph.D. degrees from the Department of Electronic and Electrical Engineering, University College Dublin, National University of Ireland, Dublin, Ireland, in 1996 and 2000, respectively.

She is currently a Post-Doctoral Research Fellow with the Department of Physical Medicine and Rehabilitation in Northwestern University at the Rehabilitation Institute of Chicago, Chicago, IL. Her research interests include mathematical modeling and analysis of bioelectric signals, in particular surface EMG.

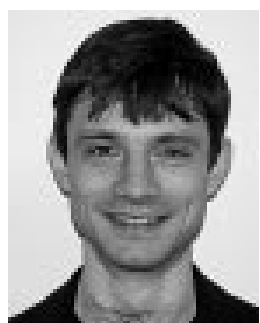

Nikolay S. Stoykov (M'00) received the M.S. and $\mathrm{Ph} . \mathrm{D}$. degrees in biomedical engineering from the Technical University Ilmenau, Ilmenau, Germany, in 1990 and 1998, respectively.

Since 1999, he has been a Post-Doctoral Fellow with the Department of Research, Rehabilitation Institute of Chicago, Chicago, IL, and with the Department of Physical Medicine and Rehabilitation, Northwestern University, Evanston, IL. His research interests have focused on modeling bioelectric phenomena with the finite-element method.

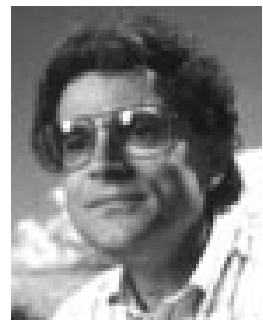

Allen Taflove (F'90) received the B.S., M.S., and $\mathrm{Ph} . \mathrm{D}$. degrees in electrical engineering from Northwestern University, Evanston, IL in 1971, 1972, and 1975, respectively.

After nine years as a Research Engineer at IIT Research Institute, Chicago, IL, he returned to Northwestern University in 1984. Since 1988, he has been a Professor in the Department of Electrical and Computer Engineering of the McCormick School of Engineering. Currently, he is a Charles Deering McCormick Professor of Teaching Excellence and Master of the Lindgren Residential College of Science and Engineering. He is conducting research in a wide range of computational electromagnetics modeling problems.

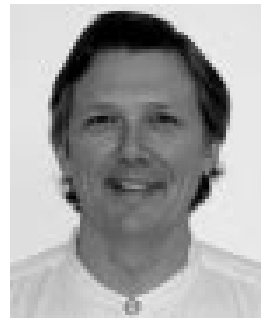

Todd A. Kuiken (M'99) received the Ph.D. degree in biomedical engineering and the M.D. degree from Northwestern University, Evanston, IL, in 1989 and 1990 , respectively. He was trained in physical medicine and rehabilitation at the Rehabilitation Institute of Chicago, Chicago, IL

$\mathrm{He}$ is currently Chief-of-Staff and the Director of Amputee Services at the Rehabilitation Institute of Chicago. He is an Assistant Professor in the Department of Physical Medicine and Rehabilitation and the Electrical and Computer Engineering Department, Northwestern University. His research interests include the care of amputees, the control of artificial limbs, and myoelectric signal analysis.

Dr. Kuiken is a board certified Physiatrist. 